\title{
Microlocalization of resonant states and estimates of the residue of the scattering amplitude
}

\author{
Jean-françois Bony Laurent Michel
}

\begin{abstract}
We obtain some microlocal estimates of the resonant states associated to a resonance $z_{0}$ of an $h$-differential operator. More precisely, we show that the normalized resonant states are $\mathcal{O}\left(\sqrt{\left|\operatorname{Im} z_{0}\right| / h}+h^{\infty}\right)$ outside the set of trapped trajectories and are $\mathcal{O}\left(h^{\infty}\right)$ in the incoming area of the phase space.

As an application, we show that the residue of the scattering amplitude of a Schrödinger operator is small in some directions under an estimate of the norm of the spectral projector. Finally we prove such bound in some examples.
\end{abstract}

\section{Introduction}

Our original motivation is the study of the residue of the scattering amplitude associate to a Schrödinger operator $P(h)=-h^{2} \Delta+V(x)$ on $\mathbb{R}^{n}$. The first works treating this question are due to Lahmar-Benbernou [11] and Lahmar-Benbernou and Martinez [12]. In these papers, they consider the case where the potential $V(x)$ is a "well in an island" with non-degenerate local minimum. In this situation, the form of the resonances is given by the work of Helffer and Sjöstrand [8]. Near a resonance $z_{0}$ simple, isolated and close to the energy of this local minimum, the scattering amplitude can be written

$$
f\left(\omega, \omega^{\prime}, z, h\right)=\frac{f^{r e s}\left(\omega, \omega^{\prime}, h\right)}{z-z_{0}}+f^{h o l}\left(\omega, \omega^{\prime}, z, h\right),
$$

with $f^{\text {hol }}$ holomorphic near $z_{0}$. Using the form of the resonant states associate to $z_{0}$, Lahmar-Benbernou and Martinez proved that

$$
\left|f^{r e s}\left(\omega, \omega^{\prime}, h\right)\right|=g(h)\left|\operatorname{Im} z_{0}\right|,
$$

where $g(h)$ has an asymptotic expansion with respect to $h$. Moreover, they showed that for some directions $\left(\omega, \omega^{\prime}\right)$, determined by the Agmon distance to the well, the

MSC 2000 : 35B34, 81Q20, 35P25..

Keywords : resonances, semiclassical analysis, scattering theory.. 
residue is $\mathcal{O}\left(h^{\infty}\right)$ while for some other ones, they obtained an explicit non-vanishing principal term for $g(h)$. Their proof is based on the knowledge of the resonant states given by [8].

In [20], Stefanov generalized some parts of this result and proved that for $V \in$ $C_{0}^{\infty}\left(\mathbb{R}^{n}\right)$ and $z_{0}$ a resonance which is simple and isolated in a sens precised in [20], one has

$$
\left|f^{r e s}\left(\omega, \omega^{\prime}, h\right)\right| \leq C h^{-\frac{n-1}{2}}\left|\operatorname{Im} z_{0}\right| .
$$

This result was next improved by the second author in [15] where estimate (1) is established for general long-range potentials under a weaker separation condition on resonances. In [20] and [15], the method employed stands on the semiclassical maximum principle of Tang and Zworski [22] and a resolvent estimate of Burq [2].

In the case where $\left|\operatorname{Im} z_{0}\right| \leq C h^{M}$ for $M \gg 1$ and $\left|\operatorname{Im} z_{0}\right| \neq \mathcal{O}\left(h^{\infty}\right)$, it proves only that $\left|f^{r e s}\right|=\mathcal{O}\left(h^{N}\right)$ for $N \in \mathbb{R}$, whereas it is proven in [12] that the decay of the residue may depend on the direction considered. In particular, one can think that there exists some couple of directions $\left(\omega, \omega^{\prime}\right)$ such that the associate residue is $\mathcal{O}\left(h^{\infty}\right)$. One of our motivations is to show the existence of such directions for resonances "far" from the real axis.

In the case where the potential $V$ is compactly supported, we have a nice representation formula for the scattering amplitude, so that one can see easily the link between the problem of the residue and the estimate of the resonant states announced in the title. Indeed, as it is proven in [16], one has

$$
f\left(\omega, \omega^{\prime}, z, h\right)=c(z ; h) \int_{\mathbb{R}^{n}} e^{-i \sqrt{z}\left\langle x, \omega^{\prime}\right\rangle / h}\left[h^{2} \Delta, \chi_{1}\right] R(z, h)\left[h^{2} \Delta, \chi_{2}\right] e^{i \sqrt{z}\langle x, \omega\rangle / h} d x
$$

where $R(z, h): L_{\text {comp }}^{2} \rightarrow H_{l o c}^{2}$ denotes the meromorphic continuation of the resolvent of $P$ to a conic neighborhood of the real axis and

$$
c(z ; h)=\frac{1}{2} z^{\frac{n-3}{4}}(2 \pi h)^{-\frac{n+1}{2}} e^{-i \frac{(n-3) \pi}{4}} .
$$

Assume that $z_{0}$ is a simple resonance and that there is no other resonance in a disk $D$ centered in $z_{0}$, then the residue is given by the formula

$$
f^{r e s}\left(\omega, \omega^{\prime}, h\right)=c\left(z_{0} ; h\right)\left\langle\left[h^{2} \Delta, \chi_{1}\right] \Pi_{\theta}\left[h^{2} \Delta, \chi_{2}\right] e^{i \sqrt{z_{0}}\langle x, \omega\rangle / h}, e^{i \sqrt{z_{0}}\left\langle x, \omega^{\prime}\right\rangle / h}\right\rangle
$$

where $\Pi_{\theta}$ is the spectral projector of $P_{\theta}$, the operator obtained from $P$ by analytic dilatation, associate to $z_{0}$. Moreover, as $\Pi_{\theta}$ is a rank one operator, there exist $u_{\theta}, v_{\theta} \in L^{2}$ such that $\Pi_{\theta}=\left\langle., v_{\theta}\right\rangle u_{\theta}$ and one can show that $\left(P_{\theta}-z_{0}\right) u_{\theta}=0$ and $\left(P_{-\theta}-\bar{z}_{0}\right) v_{\theta}=0$. It follows that

$$
f^{r e s}\left(\omega, \omega^{\prime}, h\right)=-c\left(z_{0} ; h\right)\left\langle u_{\theta},\left[h^{2} \Delta, \chi_{1}\right] e^{i \sqrt{z_{0}}\left\langle x, \omega^{\prime}\right\rangle / h}\right\rangle\left\langle\left[h^{2} \Delta, \chi_{2}\right] e^{i \sqrt{z_{0}}\langle x, \omega\rangle / h}, v_{\theta}\right\rangle .
$$

On the other hand, it is easy to see that the functions $\left[h^{2} \Delta, \chi_{*}\right] e^{i \sqrt{z_{0}}\left\langle x, \omega^{*}\right\rangle / h}$ are microlocalized near $\left\{(x, \xi) ; R_{1}<|x|<R_{2}, \xi /|\xi| \sim \omega^{*}\right\}$. Our approach consists to show that for suitable directions, the resonant sate $u_{\theta}$ is microlocalized out of this set. In fact, the microlocal estimate that we will prove holds for more general operators than Schrödinger ones. 


\section{Microlocalization of the resonant states}

In this talk, we consider $P(h)$ an $h$-differential operator on $\mathbb{R}^{n}$, having the form

$$
P(h)=\sum_{|\alpha| \leq 2} a_{\alpha}(x ; h)\left(h D_{x}\right)^{\alpha}
$$

where $a_{\alpha}(x ; h) \in S_{n}^{c l}(1)$ and $a_{\alpha}(x ; h)$ doesn't depend on $h$ for $|\alpha|=2$ (see the book of Dimassi-Sjöstrand [3] for more details on $h$-pseudodifferential calculus). We assume that $P$ is formally self-adjoint on $L^{2}\left(\mathbb{R}^{n}\right)$, that is

$$
\forall u, v \in C_{0}^{\infty}\left(\mathbb{R}^{n}\right) \quad \int(P u) \bar{v} d x=\int u \overline{(P v)} d x .
$$

We suppose also that $P$ is elliptic so that we have

$$
\sum_{|\alpha|=2} a_{\alpha}(x) \xi^{\alpha} \geq|\xi|^{2} / C
$$

To define the resonances, we assume that the coefficients $a_{\alpha}(x ; h)$ extend holomorphically in $x$ in the domain

$$
\Upsilon=\left\{x \in \mathbb{C}^{n} ;|\operatorname{Im} x| \leq \delta_{0}\langle\operatorname{Re} x\rangle \text { and }|x| \geq R_{0}\right\},
$$

$\left.R_{0}>0, \delta_{0} \in\right] 0,1\left[\right.$ and that $P$ converge to $-h^{2} \Delta$ at infinity in the following sens:

$$
\sum_{|\alpha| \leq 2} a_{\alpha}(x ; h) \xi^{\alpha} \longrightarrow \xi^{2}
$$

as $|x| \rightarrow+\infty, x \in \Upsilon$, uniformly with respect to $h$. Under these assumptions, it is clear that $P$ is a self-adjoint operator with domain $H^{2}\left(\mathbb{R}^{n}\right)$ and one can define the resonances associate to $P$ by the method of analytic distortions of Sjöstrand-Zworski [19].

Let $F: \mathbb{R}^{n} \rightarrow \mathbb{R}^{n}$ be a smooth vector field such that $F(x)=0$ if $|x| \leq R_{0}$ and $F(x)=|x|$ for $|x|$ large enough. For $\nu \in \mathbb{R}$ small enough, we consider the unitary operator $U_{\nu}$ on $L^{2}\left(\mathbb{R}^{n}\right)$ defined by:

$$
U_{\nu} \varphi(x)=\operatorname{det}(1+\nu d F(x))^{-\frac{1}{2}} \varphi(x+\nu F(x)) .
$$

Then, the operator $U_{\nu} P(h) U_{\nu}^{-1}$ has coefficients which are analytic with respect to $\nu$ near 0 and can be continued to complex values of $\nu$. For $\nu=i \theta$, with $\theta>0$ small enough, we get a differential operator denoted by $P_{\theta}$. It is well-known that the spectrum of $P_{\theta}$ is discrete in the sector $S_{\theta}=\{z \in \mathbb{C} ; \operatorname{Re} z>0$ and $-2 \theta<\arg z \leq 0\}$ (see [19]) and by definition, the resonances of $P$ are the eigenvalues of $P_{\theta}$.

We denote by $p(x, \xi ; h) \in S_{2 n}^{c l}\left(\langle\xi\rangle^{2}\right)$ the Weyl symbol of $P$ and

$$
p_{0}(x, \xi)=\sum_{|\alpha| \leq 2} a_{\alpha, 0}(x) \xi^{\alpha},
$$


is its principal symbol. The Hamilton vector field associated with $p_{0}$ is

$$
\mathrm{H}_{p_{0}}=\left(\begin{array}{c}
\partial_{\xi} p_{0} \\
-\partial_{x} p_{0}
\end{array}\right)
$$

and $\exp \left(t \mathrm{H}_{p_{0}}\right), t \in \mathbb{R}$ is the corresponding Hamiltonian flow. We define the outgoing tail and the incoming tail at the energy $E$ by

$$
\Gamma_{ \pm}(E)=\left\{(x, \xi) \in p_{0}^{-1}(E) ; \exp \left(t \mathrm{H}_{p_{0}}\right)(x, \xi) \nrightarrow \infty, t \rightarrow \mp \infty\right\},
$$

and we have the following theorem which says that a resonant state is outgoing.

Theorem 2.1 Let $E_{0}>0$ be a fixed energy level, $\epsilon>0$ small enough, $h / C<\theta<$ $C h \ln (1 / h)$ with $C>0$, let $z \in \mathbb{C}$ be a resonance of $P$ with $\operatorname{Re} z \in\left[E_{0}-\epsilon, E_{0}+\epsilon\right]$, $|\operatorname{Im} z|<\epsilon \theta$, and let $u_{\theta} \in L^{2}\left(\mathbb{R}^{n}\right)$ be a resonant state associated to $z$ :

$$
\left(P_{\theta}-z\right) u_{\theta}=0 .
$$

If $w(x, \xi) \in C_{0}^{\infty}\left(\mathbb{R}^{2 n}\right)$ with $\operatorname{supp}(w) \subset \Gamma_{+}\left(\left[E_{0}-\epsilon, E_{0}+\epsilon\right]\right)^{C}$, then for $h>0$ sufficiently small, one has

$$
\left\|w\left(x, h D_{x}\right) u_{\theta}\right\|=\mathcal{O}\left(h^{\infty}\right)\left\|u_{\theta}\right\| .
$$

Remark 2.2 It is possible to generalize this result to the black-box setting (see [19] for a precise formulation). Assume that the black-box is contained in $D\left(0, R_{0}\right)$, let $\chi \in C_{0}^{\infty}\left(\mathbb{R}^{n}\right)$ with $\chi=1$ near $D\left(0, R_{0}\right)$ and let $w$ be supported in $\left\{|x|>R_{0}\right\}$ and satisfying the assumptions of the above theorem. If $u_{\theta}$ is a resonant state, then $\left\|w\left(x, h D_{x}\right)(1-\chi(x)) u_{\theta}\right\|=\mathcal{O}\left(h^{\infty}\right)\left\|u_{\theta}\right\|$.

Before we state our second result, we introduce the set of trapped trajectories:

$$
\begin{aligned}
\mathcal{T}(E) & =\Gamma_{+}(E) \cap \Gamma_{-}(E) \\
& =\left\{(x, \xi) \in p_{0}^{-1}(E) ; t \mapsto \exp \left(t \mathrm{H}_{p_{0}}\right)(x, \xi) \text { is bounded on } \mathbb{R}\right\} .
\end{aligned}
$$

For $E>0, \mathcal{T}(E)$ is a compact set (see the appendix of the paper of C. GérardSjöstrand [7]). We give another proof of a result of Stefanov [20, 21] on the localization of the resonant states:

Theorem 2.3 Let $u_{\theta}$ be a resonant state associated to a resonance $z$ as in Theorem 2.1 with $\theta=h / C, C>0$. If $w(x, \xi) \in S_{2 n}(1)$ with $\operatorname{supp}(w) \cap \mathcal{T}\left(\left[E_{0}-\epsilon, E_{0}+\epsilon\right]\right)=\emptyset$, then

$$
\mathrm{Op}_{h}^{w}(w) u_{\theta}=\mathcal{O}\left(\sqrt{\frac{|\operatorname{Im} z|}{h}}+h^{\infty}\right)\left\|u_{\theta}\right\| .
$$

\section{Microlocal exponential estimate}

In this section, we give a microlocal exponential weighted estimate first introduced by Codoba-Fefferman. For this purpose, we use a Fourier-Bros-Iagolnitzer transform (in fact a Bargman transform), widely studied by Sjöstrand [17]. The book of Martinez [13] gives a detailed presentation. 
For $u \in \mathcal{S}^{\prime}\left(\mathbb{R}^{n}\right)$, the FBI transform of $u$ is given by

$$
T u(x, \xi ; h)=\alpha_{n}(h) \int e^{i(x-y) \xi / h-(x-y)^{2} / 2 h} u(y) d y,
$$

with $\alpha_{n}(h)=2^{-n / 2}(\pi h)^{-3 n / 4}$. It is know that $T u \in \mathcal{C}^{\infty}\left(\mathbb{R}^{2 n}\right), e^{\xi^{2} / 2 h} T u(x, \xi ; h)$ is an holomorphic function of $z=x-i \xi$ and that

$$
\|T u\|_{L^{2}\left(\mathbb{R}^{2 n}\right)}=\|u\|_{L^{2}\left(\mathbb{R}^{n}\right)} .
$$

Let $A$ be a $h$-differential operator of Weyl symbol $a(x, \xi ; h) \sim \sum_{j \geq 0} a_{j}(x, \xi) h^{j} \in$ $S_{2 n}^{c l}\left(\langle\xi\rangle^{d}\right)$. As $a$ is polynomial with respect to $\xi$ with coefficients in $S_{n}(1)$, one can find an almost analytic extension $\widetilde{a}(x, \xi ; h) \in S_{2 n}^{c l}\left(\langle\xi\rangle^{d}\right)$ of $a$ which satisfies

$$
\begin{gathered}
\widetilde{a}_{\left.\right|_{\mathbb{R}^{2 n}}}=a, \\
\partial_{\bar{x}} \widetilde{a}=\mathcal{O}\left(|\operatorname{Im} x|^{\infty}\right)\langle\xi\rangle^{d} .
\end{gathered}
$$

Following Proposition 3.1 of Martinez [13], we get

Theorem 3.1 (Martinez) Let $f(x, \xi) \in S_{2 n}(1)$ with compact support in $\xi$ and $G(x, \xi) \in C_{0}^{\infty}\left(\mathbb{R}^{2 n}\right)$. Then there exists a symbol $q(x, \xi ; t, h) \sim \sum_{j \geq 0} q_{j}(x, \xi ; t) h^{j} \in$ $S_{2 n}^{c l}(1)$ uniformly with respect to $t$ and an operator $R(t, h)$ such that for all $u, v \in$ $C_{0}^{\infty}\left(\mathbb{R}^{n}\right)$, one has

$$
\begin{aligned}
\left\langle f e^{-t G / h} T \operatorname{Op}_{h}^{w}(a) u, e^{-t G / h} T v\right\rangle_{L^{2}\left(\mathbb{R}^{2 n}\right)} & \\
& =\left\langle(q(x, \xi ; t, h)+R(t, h)) e^{-t G / h} T u, e^{-t G / h} T v\right\rangle_{L^{2}\left(\mathbb{R}^{2 n}\right)}
\end{aligned}
$$

where $\operatorname{supp} q_{j} \subset \operatorname{supp} f$ for all $j \in \mathbb{N}$,

$$
\begin{aligned}
q_{0}(x, \xi ; t)= & f(x, \xi) \widetilde{a_{0}}(x+2 t \partial z G(x, \xi), \xi-2 i t \partial z G(x, \xi)), \\
q_{1}(x, \xi ; t)= & \left(f a_{1}-f \partial_{x x}^{2} a_{0} / 4-f \partial_{\xi \xi}^{2} a_{0} / 4-\partial_{x} f \partial_{x} a_{0} / 2-\partial_{\xi} f \partial_{\xi} a_{0} / 2\right)(x, \xi) \\
& +\frac{i}{2}\left(\partial_{\xi} a_{0} \partial_{x} f-\partial_{x} a_{0} \partial_{\xi} f\right)(x, \xi)+\mathcal{O}(t)
\end{aligned}
$$

(here $\left.\partial_{z}=\left(\partial_{x}+i \partial_{\xi}\right) / 2\right)$, and

$$
\|R(t, h)\|_{\mathcal{L}\left(L^{2}\left(\mathbb{R}^{n}\right)\right)}=\mathcal{O}\left(h^{\infty}+h^{-3 n / 2}|t|^{\infty} e^{2 \sup |G||t| / h}\right),
$$

uniformly with respect to $t$ and $h$ small enough.

Theorem 3.1 is really useful for $|t|<C h \ln (1 / h)$, since, in that case, the $q_{j}$ are independent (modulo $\mathcal{O}\left(h^{\infty}\right)$ ) of the choice of the almost analytic extension $\widetilde{a}$ and $R(t, h)=\mathcal{O}\left(h^{\infty}\right)$. 


\section{Proof of Theorem 2.1}

Using the properties of $T$, it is enough to prove that

$$
w T u_{\theta}=\mathcal{O}\left(h^{\infty}\right)\left\|u_{\theta}\right\|
$$

Since $\left(P_{\theta}-z\right) u_{\theta}=0$, we can assume that $\operatorname{supp}(w) \subset p_{0}^{-1}\left(\left[E_{0}-\epsilon, E_{0}+\epsilon\right]\right)$.

$\operatorname{As} \operatorname{supp}(w) \cap \Gamma_{+}\left(\left[E_{0}-\epsilon, E_{0}+\epsilon\right]\right)=\emptyset$, we have $\exp \left(-T \mathrm{H}_{p_{0}}\right)(\operatorname{supp}(w)) \subset\{(x, \xi)$; $\langle x, \xi\rangle<-|x||\xi| / 2\}$, for $T$ large enough. Then, one can find $0 \leq \widetilde{w}(x, \xi) \in S_{2 n}$ such that $\widetilde{w}=1$ on $\exp \left(-T \mathrm{H}_{p_{0}}\right)(\operatorname{supp}(w))$ and

$$
\mathrm{H}_{p_{0}} \widetilde{w} \leq 0 .
$$

We define $\chi(x, \xi)=\widetilde{w}\left(\exp \left(T \mathrm{H}_{p_{0}}\right)(x, \xi)\right)$.

Since $p(x, \xi ; h)$ is close to $\xi^{2}$ for $x$ large $(6)$, we have, for $p_{0}(x, \xi) \in\left(\left[E_{0}-\epsilon, E_{0}+\epsilon\right]\right)$,

$$
-\operatorname{Im} p_{\theta}(x, \xi ; h) \geq \begin{cases}c \theta & \text { for }|x| \geq R \\ -M \theta & \text { for }|x| \leq R\end{cases}
$$

with $c>0$.

Now we recall the following geometric result.

Lemma 4.1 (C. Gérard-Sjöstrand [7]) Assume that $K \subset p_{0}^{-1}\left(\left[E_{0}-\epsilon, E_{0}+\epsilon\right]\right)$ is compact and satisfies $K \cap \mathcal{T}\left(\left[E_{0}-\epsilon, E_{0}+\epsilon\right]\right)=\emptyset$. Then, one can find a function $F(x, \xi) \in \mathcal{C}_{b}^{\infty}\left(\mathbb{R}^{2 n}\right)$ such that $\mathrm{H}_{p_{0}} F \geq 0$ on $p_{0}^{-1}\left(\left[E_{0}-\epsilon, E_{0}+\epsilon\right]\right)$ and $\mathrm{H}_{p_{0}} F>1$ on $K$.

We use Lemma 4.1 with $K=\operatorname{supp}(\chi) \cap D(0, R)$ and we set $G=C F$ with $C$ large enough.

Then, we apply Theorem 3.1 with $A=P_{\theta}-z$ and $t=\theta=\mathcal{O}(h \ln (1 / h))$. The inequality (13), (14) and Lemma 4.1 imply that

$$
\begin{aligned}
0 & =-\operatorname{Im}\left\langle\chi^{2}(x, \xi) e^{-t G / h} T\left(P_{\theta}-z\right) u_{\theta}, e^{-t G / h} T u_{\theta}\right\rangle \\
& \geq c \theta\left\|\chi e^{-t G / h} T u_{\theta}\right\|^{2}+\mathcal{O}\left(\theta^{2}\right)\left\|\widetilde{\chi} e^{-t G / h} T u_{\theta}\right\|^{2}+\mathcal{O}\left(h^{\infty}\right)\left\|e^{-t G / h} T u_{\theta}\right\|^{2},
\end{aligned}
$$

where $\tilde{\chi}$ satisfies the same properties as $\chi$. So

$$
\begin{aligned}
\left\|\chi e^{-t G / h} T u_{\theta}\right\|^{2} & =\mathcal{O}(\theta)\left\|\tilde{\chi} e^{-t G / h} T u_{\theta}\right\|^{2}+\mathcal{O}\left(h^{\infty}\right)\left\|e^{-t G / h} T u_{\theta}\right\|^{2} \\
& =\mathcal{O}\left(h^{\infty}\right)\left\|e^{-t G / h} T u_{\theta}\right\|^{2}
\end{aligned}
$$

by induction, and (12) follows from the fact that $e^{-t G / h}=\mathcal{O}\left(h^{-C}\right)$.

\section{Residue estimate of the scattering amplitude}

In this section, we assume that $P$ is a Schrödinger operator

$$
P=-h^{2} \Delta+V(x),
$$


where $V(x) \in S_{n}(1)$ extends holomorphically to the domain $\Upsilon$. To define the scattering amplitude, we make a long-range assumption on $V(x)$ :

$$
\exists \rho>0 \quad \exists C>0 \quad \forall x \in \Gamma, \quad|V(x)| \leq C|x|^{-\rho} .
$$

In particular, $P$ satisfies the assumptions of section 1 . We can define the scattering matrix $S(z ; h), z \in \mathbb{R}_{+}^{*}$, related to $P_{0}=-h^{2} \Delta$ and $P$, as a unitary operator:

$$
S(z ; h): L^{2}\left(\mathbb{S}^{n-1}\right) \longrightarrow L^{2}\left(\mathbb{S}^{n-1}\right) .
$$

Next, introduce the operator $T(z ; h)$ defined by $S(z ; h)=I d-2 i \pi T(z ; h)$. From Isozaki-Kitada [9], it is known that $T(z ; h)$ has a kernel $T\left(\omega, \omega^{\prime}, z ; h\right)$, smooth in $\left(\omega, \omega^{\prime}\right) \in \mathbb{S}^{n-1} \times \mathbb{S}^{n-1} \backslash\left\{\omega=\omega^{\prime}\right\}$ and the scattering amplitude is given by

$$
f\left(\omega, \omega^{\prime}, z ; h\right)=c_{1}(z ; h) T\left(\omega, \omega^{\prime}, z ; h\right),
$$

with

$$
c_{1}(z ; h)=-2 \pi(2 z)^{-\frac{n-1}{4}}(2 \pi h)^{\frac{n-1}{2}} e^{-i \frac{(n-3) \pi}{4}} .
$$

In [6], C. Gérard and Martinez have shown that for $\omega \neq \omega^{\prime}$ fixed, the scattering amplitude has a meromorphic continuation to a conic neighborhood of $\mathbb{R}_{+}^{*}$, whose poles are the resonances of $P$. Moreover, the multiplicity of each pole is exactly the multiplicity of the resonance.

In this section, we still assume that $z_{0}(h)$ is a simple resonance of $P$ such that Re $z_{0} \in\left[E_{0}-\epsilon, E_{0}+\epsilon\right]$ and $0<-\operatorname{Im} z_{0}<C h \ln (1 / h)$. Under this condition the scattering amplitude takes the form

$$
f\left(\omega, \omega^{\prime}, z ; h\right)=\frac{f^{r e s}\left(\omega, \omega^{\prime} ; h\right)}{z-z_{0}}+f^{h o l}\left(\omega, \omega^{\prime}, z ; h\right)
$$

where $f^{h o l}\left(\omega, \omega^{\prime}, z ; h\right)$ is holomorphic near $z_{0}$. Our aim is to give an estimate of the residue $f^{r e s}$ in some special directions:

Definition 5.1 We say that $\omega \in \mathbb{S}^{n-1}$ is an incoming direction (resp. outgoing direction) for the energy $E_{0}$ iff there is $\epsilon, R>0$ and $W \subset \mathbb{S}^{n-1}$, a neighborhood of $\omega$, such that, for all $(x, \xi) \in p^{-1}\left(\left[E_{0}-\epsilon, E_{0}+\epsilon\right]\right)$,

$$
|x| \geq R \text { and } \frac{\xi}{|\xi|} \in W \Longrightarrow \lim _{t \rightarrow-\infty} \exp \left(t \mathrm{H}_{p_{0}}\right)(x, \xi)=\infty \text {. }
$$

$\left(\right.$ resp. $\lim \exp \left(t \mathrm{H}_{p_{0}}\right)(x, \xi)=\infty$ as $\left.t \rightarrow+\infty\right)$

Remark 5.2 If $\rho>1, \omega$ is an incoming direction iff there is $R>0$ such that

$$
p(x, \xi)=E_{0},|x| \geq R \text { and } \frac{\xi}{|\xi|}=\omega \Longrightarrow \lim _{t \rightarrow-\infty} \exp \left(t \mathrm{H}_{p_{0}}\right)(x, \xi)=\infty .
$$

This is a consequence of the Proposition 6.1 of [14]. 
For $\theta \geq C|\operatorname{Im} z|$ with $C>0$ sufficiently large, we denote by $\Pi_{\theta}$ the spectral projector associate to the resonance $z_{0}$ :

$$
\Pi_{\theta}=\frac{1}{2 i \pi} \int_{\partial D}\left(z-P_{\theta}\right)^{-1} d z
$$

where $D=D\left(z_{0}, r(h)\right) \subset \mathbb{C}$ is a small disk such that $z_{0}$ is the only resonance in $\bar{D}$.

Theorem 5.3 Let $E_{0}>0$ and $\omega, \omega^{\prime} \in S^{n-1}$ with $\omega \neq \omega^{\prime}$. If $\omega$ is an outgoing direction or if $\omega^{\prime}$ is an incoming direction, then there exists $\epsilon, C^{\prime}>0$ such that for all simple resonance $z_{0} \in\left[E_{0}-\epsilon, E_{0}+\epsilon\right]-i\left[0, \theta / C^{\prime}\right]$ with $h / C<\theta<C h \ln (1 / h)$, $C>0$ one has

$$
f^{r e s}\left(\omega, \omega^{\prime}, h\right)=\mathcal{O}\left(h^{\infty}\right)\left\|\Pi_{\theta}\right\|
$$

Proof. We assume that $V$ has compact support and we use the formula (3). If $\omega^{\prime}$ (resp. $\omega$ ) is an incoming (resp. outgoing) direction, Theorem 2.1 implies that $u_{\theta}=$ $\mathcal{O}\left(h^{\infty}\right)\left\|u_{\theta}\right\|$ (resp. $v_{\theta}$ ) microlocally near the microsupport of $\left[h^{2} \Delta, \chi_{1}\right] e^{i \sqrt{z_{0}}\left\langle x, \omega^{\prime}\right\rangle / h}$ (resp. $\left.\left[h^{2} \Delta, \chi_{2}\right] e^{i \sqrt{z_{0}}\langle x, \omega\rangle / h}\right)$. Then

$$
f^{r e s}\left(\omega, \omega^{\prime}, h\right)=\mathcal{O}\left(h^{\infty}\right)\left\|u_{\theta}\right\|\left\|v_{\theta}\right\|=\mathcal{O}\left(h^{\infty}\right)\left\|\Pi_{\theta}\right\| .
$$

In the long range case, the proof is a little more technical since the amplitude is calculated with the method of Isozaki-Kitada.

\section{Estimate on the spectral projector}

In this section, we give some examples where the spectral projector $\Pi_{\theta}$ is bounded by $\mathcal{O}\left(h^{-C}\right)$.

- Resonances near the real axis

If the resonance $z_{0}$ satisfies $\left|\operatorname{Im} z_{0}\right|=\mathcal{O}\left(h^{M}\right)$ with $M>>1$, one can apply the semiclassical maximum principle and the a priori exponential estimate of the modified resolvent $\left(P_{\theta}-z\right)^{-1}$ obtained by Tang-Zworski [22]. Using these techniques, Stefanov [21] has proved that

Proposition 6.1 (Stefanov) Assume that $V$ is compactly supported and let $E_{0}>$ 0 . Let $z_{0}$ be a simple resonance of $P$ such that $\operatorname{Res}(P) \cap D\left(z_{0}, h^{M_{1}}\right)=\left\{z_{0}\right\}$, for $M_{1}$ sufficiently large and $\left|\operatorname{Im} z_{0}\right| \leq C h^{M_{2}}$ with $M_{2} \geq M_{1}+2 n+2$. Then

$$
\left\|\Pi_{\theta}\right\|=\mathcal{O}(1)
$$

uniformly with respect to $h / C<\theta<C h \log (1 / h)$.

- Estimate in dimension one

Proposition 6.2 We assume that $n=1$ and that the critical points of $p_{0}(x, \xi)$ on the energy level are non-degenerate (i.e. the points $(x, \xi) \in p_{0}^{-1}\left(\left\{E_{0}\right\}\right)$ such that 
$\nabla p_{0}(x, \xi)=0$ satisfy Hess $p_{0}(x, \xi)$ is invertible $)$. Then there exists $M, \epsilon>0$ such that, for $E \in\left[E_{0}-\epsilon, E_{0}+\epsilon\right]$ and $\theta=N h$ with $N>0$ large enough,

$$
\left\|\left(P_{\theta}-z\right)^{-1}\right\|=\mathcal{O}\left(h^{-M}\right) \prod_{z_{j} \in \operatorname{Res}(P) \cap \Omega_{E, \epsilon \theta}} \frac{\theta}{\left|z-z_{j}\right|},
$$

where $z \in \Omega_{E, \epsilon \theta / 2}, \Omega_{E, \delta}=E+D(0, \delta)$ and $h$ is small enough.

Proof. The proof is based on the a priori estimate of the resolvent given by Tang-Zworski [22] and on the fact that the number of resonances in $D\left(E_{0}, C h\right)$ is $\mathcal{O}\left(\ln (1 / h) h^{1-n}\right)=\mathcal{O}(\ln (1 / h))$.

As a direct consequence, we get

Corollary 6.3 Under the hypotheses of Lemma 6.2, if \#Res $(P) \cap D\left(E_{0}, \theta\right)=\mathcal{O}(1)$ and $z_{0} \in \operatorname{Res}(P)$ is separated by $h^{C}$ from the other resonances of $P$, then

$$
\Pi_{\theta}=\mathcal{O}\left(h^{-C^{\prime}}\right) .
$$

We can also study the following example. Consider a short range potential $V(x)$ which is holomorphic in

$$
\{x \in \mathbb{C} ;|\operatorname{Im} z| \leq\langle\operatorname{Re} z\rangle / C\}
$$

and has the following form:

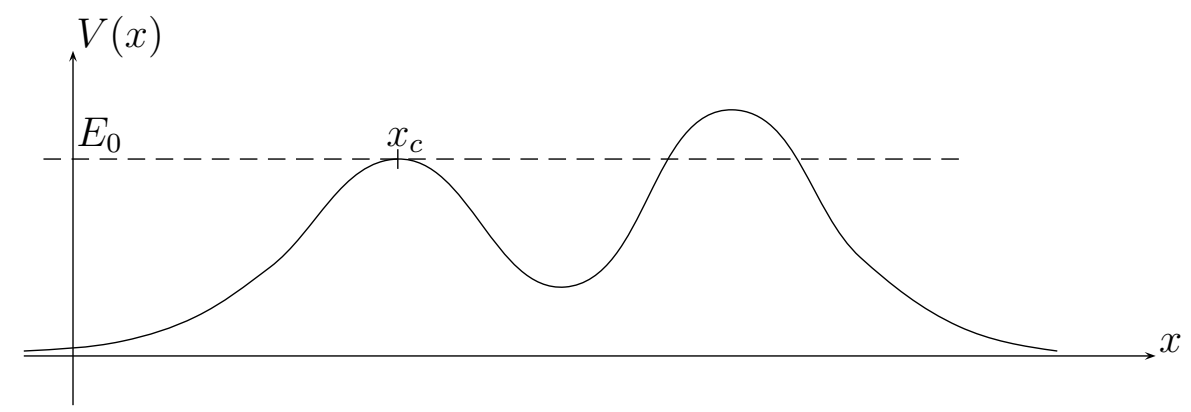

At $x_{c}, V(x)$ has a non degenerate maximum. Such type of potential have been study by Fujiié and Ramond [4] and [5]. In particular, the formula (41) of [5] implies that the resonances in $\Omega_{E_{0}, \epsilon \theta}$ are of the form

$$
z_{j}=E_{0}+\frac{S_{0}-(2 j+1) \pi h+i h \ln (2)}{K \ln (h)}+\mathcal{O}\left(h / \ln (h)^{2}\right),
$$

with $j \in \mathbb{Z}$ and $S_{0}, K$ are some fixed constants.

Corollary 6.4 Under the previous hypotheses, the projector associated to a resonance $z_{j}$ satisfies, for $h$ small enough,

$$
\Pi_{\theta}=\mathcal{O}\left(h^{-C}\right) .
$$

In this case $+1 \in \mathbb{S}^{0}$ is an incoming direction and -1 is an outgoing direction. 
- Estimate near critical energy

Let $P=-h^{2} \Delta+V(x)$ where $V(x)$ is a short range potential which is holomorphic in

$$
\left\{x \in \mathbb{C}^{n} ;|\operatorname{Im} z| \leq\langle\operatorname{Re} z\rangle / C\right\} .
$$

We assume that $V(x)$ has a non-degenerate critical point at $x_{c}$ at the energy $E_{0}$ (i.e. $V\left(x_{c}\right)=E_{0}, V^{\prime}\left(x_{c}\right)=0$ and $V^{\prime \prime}\left(x_{c}\right)$ is invertible with signature $\left(p_{+}, p_{-}\right)$) and $\mathcal{T}\left(E_{0}\right)=\left\{\left(x_{0}, 0\right)\right\}$. In this situation Sjöstrand [18] described all the resonances in $D\left(E_{0}, C h\right)$. In particular, if the $\lambda_{j}$ are $\mathbb{Z}$-independent, we know that the distance between two resonances in this set is of order $h$.

Under these hypotheses, the center-stable manifolds Theorem (see [1, Theorem 7.2.2]) gives $\Lambda_{+} \subset \Gamma_{-}\left(E_{0}\right)^{c}$, (resp. $\left.\Lambda_{-} \subset \Gamma_{+}\left(E_{0}\right)^{c}\right) p_{+}$-dimensional manifold passing through the origin and invariant under the $\mathrm{H}_{p_{0}}$-flow. Moreover, $\omega \in \mathbb{S}^{n-1}$ is not an incoming (resp. outgoing) direction iff there is $(x(t), \xi(t))=\exp \left(t \mathrm{H}_{p_{0}}\right)(x, \xi) \in \Lambda_{+}$ (resp. $\Lambda_{-}$) such that $\xi(t) /|\xi(t)| \rightarrow \omega$ as $t \rightarrow+\infty$.

For instance, in dimension 2, for the following potential:

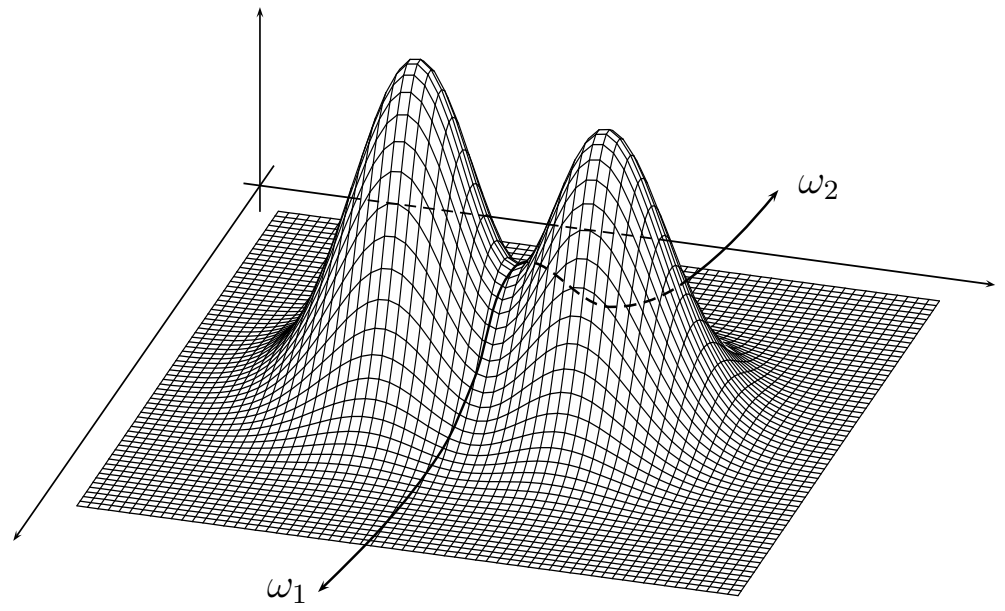

the incoming (resp. outgoing) directions are $\mathbb{S}^{1} \backslash\left\{\omega_{1}, \omega_{2}\right\}$ (resp. $\mathbb{S}^{1} \backslash\left\{-\omega_{1},-\omega_{2}\right\}$ ).

Proposition 6.5 Let $z_{0} \in D\left(E_{0}, C h\right), C>0$, be a resonance of $P$ separated by $h^{C}$ from the other resonances. For $\theta=C^{\prime} h \ln (1 / h), C^{\prime}>0$, the projector associated to $z$ satisfies

$$
\Pi_{\theta}=\mathcal{O}\left(h^{-C^{\prime \prime}}\right)
$$

Proof. This is a slight modification of some parts of the works of Sjöstrand [18] and Kaidi-Kerdelhué [10].

\section{References}

[1] R. Abraham and J. E. Marsden, Foundations of mechanics, Second edition, Advanced Book Program, Benjamin/Cummings Publishing, 1978.

[2] N. Burq, Lower bounds for shape resonances widths of long range Schrödinger operators, Amer. J. Math. 124 (2002), no. 4, 677-735. 
[3] M. Dimassi and J. Sjöstrand, Spectral asymptotics in the semi-classical limit, Cambridge University Press, Cambridge, 1999.

[4] S. Fujiié and T. Ramond, Matrice de scattering et résonances associées à une orbite hétérocline, Ann. Inst. H. Poincaré Phys. Théor. 69 (1998), no. 1, 31-82.

[5] S. Fujiié and T. Ramond, Breit-wigner formula at barrier tops, preprint (2002).

[6] C. Gérard and A. Martinez, Prolongement méromorphe de la matrice de scattering pour des problèmes à deux corps à longue portée, Ann. Inst. H. Poincaré Phys. Théor. 51 (1989), no. 1, 81-110.

[7] C. Gérard and J. Sjöstrand, Semiclassical resonances generated by a closed trajectory of hyperbolic type, Comm. Math. Phys. 108 (1987), no. 3, 391-421.

[8] B. Helffer and J. Sjöstrand, Résonances en limite semi-classique, Mém. Soc. Math. France (N.S.) (1986), no. 24-25.

[9] H. Isozaki and H. Kitada, Scattering matrices for two-body schrödinger operators, Sci. Papers College Arts Sci. Univ Tokyo 35 (1985), no. 1, 81-107.

[10] N. Kaidi and P. Kerdelhué, Forme normale de Birkhoff et résonances, Asymptot. Anal. 23 (2000), no. 1, 1-21.

[11] A. Lahmar-Benbernou, Estimation des résidus de la matrice de diffusion associés à des résonances de forme. I, Ann. Inst. H. Poincaré Phys. Théor. 71 (1999), no. 3, 303-338.

[12] A. Lahmar-Benbernou and A. Martinez, Semiclassical asymptotics of the residues of the scattering matrix for shape resonances, Asymptot. Anal. 20 (1999), no. 1, 13-38.

[13] A. Martinez, An introduction to semiclassical and microlocal analysis, SpringerVerlag, New York, 2002.

[14] L. Michel, Semi-classical behavior of the scattering amplitude for trapping perturbations at fixed energy, Can. J. Math., to appear.

[15] L. Michel, Semi-classical estimate of the residue of the scattering amplitude for long-range potentials, J. Phys. A 36 (2003), 4375-4393.

[16] V. Petkov and M. Zworski, Semi-classical estimates on the scattering determinant, Ann. Henri Poincaré 2 (2001), no. 4, 675-711.

[17] J. Sjöstrand, Singularités analytiques microlocales, Astérisque, 95, Astérisque, vol. 95, Soc. Math. France, Paris, 1982, pp. 1-166.

[18] J. Sjöstrand, Semiclassical resonances generated by nondegenerate critical points, Pseudodifferential operators (Oberwolfach, 1986), Springer, Berlin, 1987, pp. 402-429. 
[19] J. Sjöstrand and M. Zworski, Complex scaling and the distribution of scattering poles, J. Amer. Math. Soc. 4 (1991), no. 4, 729-769.

[20] P. Stefanov, Estimates on the residue of the scattering amplitude, Asympt. Anal. 32 (2002), no. 3,4, 317-333.

[21] P. Stefanov, Sharp upper bounds on the number of resonances near the real axis for trapped systems, Amer. J. Math., 125 (2003), no. 1, 183-224.

[22] S-H. Tang and M. Zworski, From quasimodes to reasonances, Math. Res. Lett. 5 (1998), no. 3, 261-272.

Mathématiques Appliquées de Bordeaux, UMR 5466 du CNRS UNIVERSITÉ DE BORDEAUX 1

33405 TALENCE CEDEX

FRANCE

bony@math .u-bordeaux.fr

lmichel@math.u-bordeaux.fr 